\title{
Prioritization of Deleterious Variations in the Fuman Hypoxanthine-Guanine Phosphoribosyltransferase Gene
}

\author{
Semira Kheiri (MSc) \\ Department of Biotechnology, \\ Laboratory Sciences Research Center, \\ Golestan University of Medical \\ Sciences, Gorgan, Iran \\ Mahdieh Safarzad (MSc) \\ Department of Biochemistry, \\ Laboratory Sciences Research Center, \\ Golestan University of Medical \\ Sciences, Gorgan, Iran \\ Mohammad Shariati (BSc) \\ Department of Microbiology, \\ Laboratory of Biotechnology, School \\ of Advanced Technologies in \\ Medicine, Golestan University of \\ Medical Sciences, Gorgan, Iran \\ Hoda Sohrabi (MSc) \\ Department of Medical Biotechnology, \\ Department of Medical Biotechnology, \\ School of Advanced Technologies in \\ Medicine, Golestan University of \\ Medical Sciences, Gorgan, Iran \\ Corresponding author: Hoda Sohrabi \\ E-mail: hsohrabi65@yahoo.com \\ Tel: $+98-9111780406$ \\ Address: Golestan University of \\ Medical Sciences, Gorgan, Iran \\ Received : 28 Sep 2016 \\ Revised: 09 Aug 2016 \\ Accepted: 11 Aug 2016
}

\begin{abstract}
Background and Objectives: Non-synonymous single nucleotide polymorphisms are typical genetic variations that may potentially affect the structure or function of expressed proteins, and therefore could be involved in complex disorders. A computationalbased analysis has been done to evaluate the phenotypic effect of non-synonymous single nucleotide polymorphisms in the gene encoding the human hypoxanthine-guanine phosphoribosyltransferase (HGPRT-1). HGPRT-1 is an enzyme involved in purine recycling pathway and its deficiency is associated with several human genetic disorders.

Methods: We provide a list of all amino acid replacements in the human HGPRT-1 from the dbSNP, Uniprot and dbEST databases. Sorting intolerant from tolerant (SIFT) and PolyPhen softwares were also used in our study.

Results: 0f 94 amino acid substitutions, rs 267606063 was predicted to be the most deleterious. Substitutions of S110L and S104A in flexible loop and D194N, D201Y, H204R, Y195C, F199V and H204D in hood domain were predicted as functionally damaging.

Conclusion: It could be concluded that these intolerant changes may lie within a functional region of the protein and may affect the stability and folding of HGPRT-1. These variants could be used for future functional and molecular epidemiology studies of HCPRT-1related disorders.
\end{abstract}

Keywords: Polymorphism, Single Nucleotide, Amino acid substitution, Hypoxanthine Phosphoribosyltransferase. 


\section{INTRODUCTION}

Hypoxanthine-guaninephosphoribosyl transferase-1 (HGPRT-1) is an enzyme associated with Lesch-Nyhan syndrome (LNS), which leads to hyperuricemia $(1,2)$. HGPRT-1 deficiency may also cause megaloblastic anemia. This enzyme plays a critical role in purine salvage pathway and catalyzes the synthesis of guanosine monophosphate and inosine monophosphate from guanine and hypoxanthine, respectively (3). Moreover, knockout of HGPRT-1 in mice inhibited the increase of dopamine in brain, but no neurological alterations have been shown (4). Moreover, uric acid overproduction is present in all HPRT-deficient patients and is associated with lithiasis and gout (5). Single nucleotide polymorphisms (SNPs) are known as the most common changes in the human genome, however only a small group of these variations can be considered as markers of susceptibility to various common human diseases (6). However, understanding the functions of coding substitutions can noticeably help understand the genetics of the human phenotypic variations. Among these alterations, non-synonymous (ns) SNPs, especially missense SNPs that cause amino acid substitutions in the encoded protein sequence are thought to have significant deleterious effects on the structure and/or function of the related protein $(7,8)$. Experimental analysis of all functionally important replacements is difficult. Predicting the deleterious variants for a key gene, such as HGPRT-1, from the majority of benign (no observable phenotypic impact) variants has been receiving much attention. Thus, computational strategies based on the biochemical severity of the amino acid substitution and protein sequence and/or structural information are proposed for the systematic analysis of genetic alterations (6, $9)$. In this study, we predicted variations of the HGPRT-1 gene that potentially affect its protein function.

\section{MATERIAL AND METHODS}

The nsSNPs in the HGPRT-1 gene and related protein sequences were retrieved from the National Center for Biotechnology Information (NCBI) database of SNPs, dbSNP (http://www.ncbi.nlm.-nih.gov/projects/SNP) and Uniprot (http://www.uniprot.org) for the computational analysis. To assess amino acid substitutions in HGPRT-1, we compared the amino acid sequence of HGPRT-1 (NP_000185.1) against the most recent EST database (build 130) using the tBlastn algorithm

(http://www.ncbi.nlm.nih.gov/BLAST/) (1012). Overall, 110 HGPRT-1 or HGPRT-1related sequences were screened. Only variants that were observed more than once were scored as possible amino acid substitutions and the rest were not included in the study. In addition, nonsense $(*)$ or ambiguous $(\mathrm{B}, \mathrm{Z}, \mathrm{X}$, etc.) amino acid substitutions were not considered in the analysis.

Sorting intolerant from tolerant (SIFT) is based on a premise that protein evolution is correlated with protein function. SIFT is a sequence homology-based tool that sorts intolerant from tolerant amino acid substitutions and predicts whether an amino acid substitution in a protein will have a phenotypic effect (13). We used SIFT to detect the deleterious coding variants and submitted the query in the form of either SNP IDs or as protein sequences. SIFT analysis was performed by allowing the algorithm to search for homologous sequences using the default settings (SWISS-PROT 45 and TrEMBL 28 databases, median conservation score 3.00, remove sequences $>90 \%$ identical to query sequence). The tool generates alignments with a large number of homologous sequences and assigns scores to each residue, ranging from 0 to 1 . Scores close to 0 indicate evolutionary conservation and intolerance to substitution, while scores close to 1 indicate tolerance to substitution. SIFT scores $<0.05$ are predicted by the algorithm to be intolerant or deleterious amino acid substitutions, whereas scores $>0.05$ are considered tolerant. The higher the tolerance index of a particular amino acid substitution, the lesser is its probable impact. Polymorphism phenotyping (PolyPhen) is an automatic tool for prediction of the possible impact of an amino acid substitution on the structure and function of a human protein (14). This prediction is based on straightforward empirical rules that are applied to the sequence and phylogenetic and structural information SWALL database ID or accession number together with sequence position with two amino acid alterations. We submitted the 
identifying the substitution. Input options for the PolyPhen server are protein sequence, query in the form of protein sequence with mutational position and two amino acid variants.

PolyPhen searches for 3D protein structures, multiple alignments of homologous sequences and amino acid information in several protein structure databases. Then, it calculates position-specific independent count (PSIC) scores for each of the two variants and computes the difference of the PSIC scores of the two variants.

Higher PSIC score differences indicate greater functional impact of a particular amino acid substitution. PolyPhen scores were designated as "probably damaging" (0.95-1), "possibly damaging" (0.7-0.95), and "benign" (0.00$0.31)$.

\section{RESULTS}

The human HGPRT-1 gene retrieved from the dbSNP, dbEST and Uniprot databases contained 94 coding variants. Corresponding information for the variants including allele changes, amino acid substitutions and substitution positions are listed in table 1. In the PolyPhen analysis, protein sequences with mutational position and amino acid variants were submitted as inputs to the PolyPhen. This algorithm predicted 36 (38.2\%) of these variants to be probably damaging, $19(20.2 \%)$ to be possibly damaging and $39(41.4 \%)$ to be benign substitutions. Moreover, all studied variants were predicted to be absolutely tolerated by the SIFT software (SIFT score= 1). The software showed low confidence that means the protein alignment does not have enough sequence diversity.

Table 1- SIFT and Polyphen predictions for nsSNPs in the human HGPRT1 gene

\begin{tabular}{|c|c|c|c|c|c|}
\hline \#rs ID & Allele change & Amino acid substitution & $\begin{array}{l}\text { substitution } \\
\text { position }\end{array}$ & PolyPhen prediction & PolyPhen score \\
\hline rs137852477 & ATT $\Rightarrow$ ATG & I $[$ Ile] $\Rightarrow$ M $[$ Met $]$ & 132 & Possibly damaging & 0.930 \\
\hline rs137852478 & GAT $\Rightarrow$ GTT & $\mathrm{D}[\mathrm{Asp}] \Rightarrow \mathrm{V}[\mathrm{Val}]$ & 80 & Possibly damaging & 0.732 \\
\hline rs137852479 & GAT $\Rightarrow$ GGT & D $[$ Asp $] \Rightarrow$ G $[$ Gly $]$ & 201 & Possibly damaging & 0.951 \\
\hline rs137852480 & $\mathrm{CTA} \Rightarrow \mathrm{CCA}$ & $\mathrm{L}[\mathrm{Leu}] \Rightarrow \mathbf{P}[$ Pro $]$ & 41 & probably damaging & 1.000 \\
\hline rs137852481 & TTC $\Rightarrow$ TTA & F $[$ Phe $] \Rightarrow$ L [Leu $]$ & 74 & probably damaging & 0.999 \\
\hline rs137852482 & TCA $\Rightarrow$ TTA & $\mathrm{S}[\mathrm{Ser}] \Rightarrow \mathrm{L}[\mathrm{Leu}]$ & 110 & probably damaging & 0.987 \\
\hline rs137852483 & GTC $\Rightarrow$ GAC & $\mathrm{V}[\mathrm{Val}] \Rightarrow \mathrm{D}[\mathrm{Asp}]$ & 130 & probably damaging & 0.978 \\
\hline rs137852484 & GCA $\Rightarrow$ TCA & $\mathrm{A}[\mathrm{Ala}] \Rightarrow \mathrm{S}[\mathrm{Ser}]$ & 161 & Possibly damaging & 0.905 \\
\hline rs137852485 & AGC $\Rightarrow$ AGA & $\mathbf{S}[\mathrm{Ser}] \Rightarrow \mathbf{R}[\mathbf{A r g}]$ & 104 & probably damaging & 1.000 \\
\hline rs137852486 & TTC $\Rightarrow$ GTC & F $[$ Phe $] \Rightarrow$ V [Val] & 199 & probably damaging & 0.985 \\
\hline rs137852487 & GGG $\Rightarrow$ GAG & G $[\mathrm{Gly}] \Rightarrow \mathrm{E}[\mathrm{Glu}]$ & 70 & probably damaging & 1.000 \\
\hline rs137852488 & GGC $\Rightarrow$ CGC & G $[\mathrm{Gly}] \Rightarrow R[\mathrm{Arg}]$ & 71 & probably damaging & 0.971 \\
\hline rs137852489 & $\mathbf{C A G} \Rightarrow \mathbf{A A G}$ & $Q[\mathbf{G l n}] \Rightarrow \mathrm{K}[\mathrm{Lys}]$ & 109 & Benign & 0.001 \\
\hline rs137852490 & $\mathrm{CAT} \Rightarrow \mathrm{GAT}$ & $\mathbf{H}[\mathrm{His}] \Rightarrow \mathrm{D}[\mathrm{Asp}]$ & 204 & Possibly damaging & 0.747 \\
\hline rs137852491 & AGG $\Rightarrow$ AAG & $R[\operatorname{Arg}] \Rightarrow K[$ Lys $]$ & 45 & probably damaging & 1.000 \\
\hline rs137852492 & GAC $\Rightarrow$ TAC & $D[$ Asp $] \Rightarrow Y[T y r]$ & 177 & probably damaging & 1.000 \\
\hline rs137852493 & $\mathrm{CCA} \Rightarrow \mathrm{CTA}$ & $P[$ Pro $] \Rightarrow L[$ Leu $]$ & 176 & probably damaging & 0.994 \\
\hline rs137852494 & CGA $\Rightarrow$ GGA & $R[\operatorname{Arg}] \Rightarrow$ G [Gly $]$ & 51 & Benign & 0.081 \\
\hline rs137852495 & ATG $\Rightarrow$ ACG & M $[$ Met $] \Rightarrow$ T $[$ Thr $]$ & 57 & Benign & 0.009 \\
\hline rs137852496 & ATG $\Rightarrow$ AAG & $M[$ Met $] \Rightarrow$ K $[$ Lys $]$ & 143 & probably damaging & 1.000 \\
\hline rs137852498 & $\mathrm{ACC} \Rightarrow \mathrm{ATC}$ & $\mathrm{T}[\mathrm{Thr}] \Rightarrow \mathrm{I}[\mathrm{Ile}]$ & 168 & Benign & 0.258 \\
\hline rs137852499 & GGT $\Rightarrow$ AGT & G $[\mathrm{Gly}] \Rightarrow \mathrm{S}[\mathrm{Ser}]$ & 16 & Possibly damaging & 0.792 \\
\hline rs137852500 & GGA $\Rightarrow$ AGA & G $[\mathrm{Gly}] \Rightarrow R[\mathrm{Arg}]$ & 58 & Benign & 0.029 \\
\hline rs137852501 & CTG $\Rightarrow$ GTG & $\mathbf{L}[\mathbf{L e u}] \Rightarrow \mathbf{V}[$ Val $]$ & 78 & probably damaging & 0.961 \\
\hline rs137852502 & GAT $\Rightarrow$ GGT & $\mathbf{D}[$ Asp $] \Rightarrow$ G $[\mathrm{Gly}]$ & 52 & Possibly damaging & 0.869 \\
\hline rs137852503 & GGC $\Rightarrow$ GAC & G $[\mathrm{Gly}] \Rightarrow \mathrm{D}[\mathrm{Asp}]$ & 140 & probably damaging & 1.000 \\
\hline rs137852504 & GAC $\Rightarrow$ GAG & $\mathbf{D}[\mathbf{A s p}] \Rightarrow \mathrm{E}[\mathrm{Glu}]$ & 194 & Possibly damaging & 0.520 \\
\hline rs137852506 & $\mathrm{CTC} \Rightarrow \mathrm{TTC}$ & L $[$ Leu $] \Rightarrow$ F [Phe $]$ & 65 & probably damaging & 0.999 \\
\hline rs267606863 & $\mathbf{G A C} \Rightarrow \mathbf{A A C}$ & $\mathbf{D}[\mathbf{A s p}] \Rightarrow \mathbf{N}[\mathbf{A s n}]$ & 194 & probably damaging & 0.973 \\
\hline rs387906725 & $\mathrm{CGT} \Rightarrow \mathrm{CAT}$ & $\mathbf{R}[\mathrm{Arg}] \Rightarrow \mathbf{H}[\mathrm{His}]$ & 48 & probably damaging & 1.000 \\
\hline rs398123242 & $\mathbf{A A A} \Rightarrow \mathbf{G A A}$ & $\mathrm{K}[\mathrm{Lys}] \Rightarrow \mathrm{E}[\mathrm{Glu}]$ & 166 & Possibly damaging & 0.790 \\
\hline rs369065223 & TCA $\Rightarrow$ TTA & $\mathrm{S}[\mathrm{Ser}] \Rightarrow \mathrm{L}[\mathrm{Leu}]$ & 123 & Benign & 0.047 \\
\hline rs371800609 & $\mathrm{CGA} \Rightarrow \mathrm{CAA}$ & $R[\operatorname{Arg}] \Rightarrow Q[G \mid n]$ & 51 & Benign & 0.059 \\
\hline rs374646638 & GAC $\Rightarrow$ GAA & $\mathrm{D}[\mathrm{Asp}] \Rightarrow \mathrm{E}[\mathrm{Glu}]$ & 18 & Benign & 0.000 \\
\hline rs534390401 & $\mathrm{CCA} \Rightarrow \mathrm{GCA}$ & $\mathbf{P}[\mathrm{Pro}] \Rightarrow \mathbf{A}[$ Ala $]$ & 169 & Benign & 0.006 \\
\hline
\end{tabular}




\section{DISCUSSION}

HGPRT-1 is an important enzyme that has a key role in purine recycling pathway. Numerous investigations showed that alterations in the HGPRT- 1 gene may affect its phenotype in several human disorders $(15,16)$. LNS is a rare $\mathrm{X}$-linked recessive inherited neurogenetic disorder caused by deletion of exons 2 and 3 of the HGPRT gene, which results in deficiency of the purine salvage enzyme (HGPRT) $(17,18)$. The human HGPRT-1 gene is located in the q26-q27 region of the $\mathrm{X}$ chromosome and has eight introns and nine exons $(19,20)$. The coding region of this gene contains 654 nucleotides encoding a core domain containing the phosphoribosylpyrophosphate (PRPP) binding site and a hood domain (residues 188-204) that binds to the purine base substrate $(21,22)$. Results of the PolyPhen analysis suggested that amino acid substitutions S110L and S104A located at the flexible loop (residues 101-111), which is involved in interactions with the bound $\mathrm{Mg}^{2+}$ PRPP molecule and F199V, H204D, D194N, D201Y, H204R and Y195C in the hood domain contributing to the purine base binding may be highly deleterious and affect the protein function. According to SIFT, amino acid changes at surface of this protein probably have less structural and functional effects because all the studied alterations have been absolutely tolerated $($ score $=1.00)$ and the role of evolutionary players may interpret these observations. Furthermore, among the damaging replacements, only D194N (rs267606863) occurs at the $\mathrm{Mg}^{2+}$ binding site of the enzyme that has been determined to be a highly effective substitution on the structure of this protein. Considering the diversity of rules for predicting the effect of alterations on protein function, the outcomes could be somewhat dissimilar. However, the most reliable result is predictions of an SNP that overlap in various algorithms. There is no direct approach to evaluate the accuracy of these predictions made by SIFT and PolyPhen, as it is possible that the algorithms may have used different data sets (23). Hence, even if the predictions made by these softwares were not completely consistent for this subset of mutations, these HGPRT-1 variants should still be regarded as candidates for SNP screening. Furthermore, molecular modeling and experimental investigations can be used to confirm our results and clarify possible regulatory functions of the studied deleterious replacements.

\section{CONCLUSION}

Screening of the HGPRT-1 gene mutations revealed the existence of many polymorphic sites, some of which are associated with phenotypic traits and diseases risk. However, given the high number of SNPs in this gene, association studies should be carried on the genetic variants with functional significance. Use of computational tools may be an alternative approach to select functional SNPs. Furthermore, molecular modeling and experimental investigations can be used to confirm our results and clarify possible regulatory functions of the studied deleterious replacements.

\section{ACKNOWLEDGMENTS}

This study received approval from the Golestan University of Medical Sciences, Iran. We would like to thank our colleagues, especially Mr. Esmaeil Samadian for useful comments.

\section{CONFLICT OF INTEREST}

The authors declare that there is no conflict of interest. 


\section{REFERENCES}

1. Hladnik U, Nyhan WL, Bertelli M. Variable expression of HPRT deficiency in 5 members of a family with the same mutation. Archives of neurology. 2008; 65(9): 1240-3. doi: 10.1001/archneur.65.9.1240.

2. Chandekar P, Madke B, Kar S, Yadav N. Lesch-nyhan syndrome in an Indian child. Indian journal of dermatology. 2015; 60(3): 298-300. doi: 10.4103/00195154.156392.

3. Torres RJ, Puig JG. Hypoxanthine-guanine phosophoribosyltransferase (HPRT) deficiency: LeschNyhan syndrome. Orphanet journal of rare diseases. 2007; 2 (1): 48. doi.org/10.1186/1750-1172-2-48.

4. Engle SJ, Womer DE, Davies PM, Boivin G, Sahota A, Simmonds HA, et al. HPRT-APRT-deficient mice are not a model for Lesch-Nyhan syndrome. Human molecular genetics. 1996; 5(10): 1607-10.

5. Torres RJ, Prior C, Puig JG. Efficacy and safety of allopurinol in patients with hypoxanthine-guanine phosphoribosyltransferase deficiency. Metabolism. 2007; 56(9): 1179-86.

6. Wang L-L, Li Y, Zhou S-F. Prediction of deleterious non-synonymous single nucleotide polymorphisms of genes related to ethanol-induced toxicity. Toxicology letters. 2009; 187(2): 99-114. doi: 10.1016/j.toxlet.2009.02.007.

7. Bao L, Cui Y. Functional impacts of non-synonymous single nucleotide polymorphisms: Selective constraint and structural environments. FEBS letters. 2006; 580(5): 1231-4. DOI:10.1016/j.febslet.2006.01.035.

8. Chasman D, Adams RM. Predicting the functional consequences of non-synonymous single nucleotide polymorphisms: structure-based assessment of amino acid variation. Journal of molecular biology. 2001;307(2):683-706. DOI:10.1006/jmbi.2001.4510.

9. Jordan DM, Ramensky VE, Sunyaev SR. Human allelic variation: perspective from protein function, structure, and evolution. Current opinion in structural biology. 2010; 20(3): $342-50$. doi: 10.1016/j.sbi.2010.03.006.

10. Altschul SF, Gish W, Miller W, Myers EW, Lipman DJ. Basic local alignment search tool. Journal of molecular biology. 1990; 215(3): 403-10. DOI:10.1016/S0022-2836(05)80360-2.

11. Gish W, States DJ. Identification of protein coding regions by database similarity search. Nature genetics. 1993; 3(3): 266-72.

12. Boguski MS, Lowe TM, Tolstoshev CM. dbESTdatabase for "expressed sequence tags". Nature genetics. 1993; 4(4): 332-3.
13. Ng PC, Henikoff S. SIFT: Predicting amino acid changes that affect protein function. Nucleic acids research. 2003; 31(13): 3812-4.

14. Ramensky V, Bork P, Sunyaev S. Human nonsynonymous SNPs: server and survey. Nucleic acids research. 2002; 30(17): 3894-900.

15. Fu R, Ceballos-Picot I, Torres RJ, Larovere LE, Yamada Y, Nguyen KV, et al. Genotype-phenotype correlations in neurogenetics: Lesch-Nyhan disease as a model disorder. Brain. 2014;137(5):1282-303. doi: 10.1093/brain/awt202.

16. Monzani PS, Trapani S, Thiemann OH, Oliva G. Crystal structure of Leishmania tarentolae hypoxanthineguanine phosphoribosyltransferase. BMC structural biology. 2007;7(1): 59.

17. Baba S, Saito T, Yamada Y, Takeshita E, Nomura N, Yamada $\mathrm{K}$, et al. Novel mutation in HPRT1 causing a splicing error with multiple variations. Nucleosides, Nucleotides and Nucleic Acids. 2017; 36(1): 1-6. doi: 10.1080/15257770.2016.1163381.

18. Thumfart J, Weschke B, Ringe H, Weinhold N, Müller D. Acute renal failure unmasking Lesch-Nyhan disease in a patient with tuberous sclerosis complex. European Journal of Paediatric Neurology. 2016; 20(4): 649-51. doi: 10.1016/j.ejpn.2016.04.014.

19. Keebaugh AC, Sullivan RT, Thomas JW, Program NCS. Gene duplication and inactivation in the HPRT gene family. Genomics. 2007; 89(1): 134-42. DOI:10.1016/j.ygeno.2006.07.003.

20. Flier JS, Moore MJ, Wilson JM, Young AB, Kelley WN. Hypoxanthine-guanine phosphoribosyltransferase deficiency: The molecular basis of the clinical syndromes. New England Journal of Medicine. 1983; 309(15): 900-10.

21. Duan J, Nilsson L, Lambert B. Structural and functional analysis of mutations at the human hypoxanthine phosphoribosyl transferase (HPRT1) locus. Human mutation. 2004; 23(6): 599-611.

22. Balendiran GK, Molina J, Xu Y, Torres-Martinez J, Stevens R, Focia PJ, et al. Ternary complex structure of human HGPRTase, PRPP, Mg2+, and the inhibitor HPP reveals the involvement of the flexible loop in substrate binding. Protein Science. 1999; 8(5): 1023-31. DOI: $10.1110 /$ ps.8.5.1023.

23. Naderi M, Gharaei R, Soleymani-Nejadian E, Samadian E. In Silico survey of functional coding variants in human AEG-1 gene. Egyptian Journal of Medical Human Genetics. 2013;14(4):419-22. 